\title{
Quantum preequilibrium multistep direct calculations for nucleon scattering on spherical and deformed nuclei: a microscopic approach
}

\author{
Marc Dupuis $^{1}$, Ludovic Bonneau ${ }^{1}$, Toshihiko Kawano ${ }^{1}$, Eric Bauge ${ }^{2}$, Jean Paul Delaroche ${ }^{2}$, and Daniel Gogny ${ }^{3}$ \\ 1 T-16 Nuclear Physics, Los Alamos National Laboratory, New Mexico, USA \\ 2 CEA/DAM Île-de-France, DPTA/SPN, Bruyères-le-Châtel, France \\ 3 Lawrence Livermore National Laboratory, California, USA
}

\begin{abstract}
Preequilibrium multistep direct (MSD) double differential cross sections are calculated for medium energy inelastic neutron scattering $(11-18 \mathrm{MeV})$ on ${ }^{208} \mathrm{~Pb}$ and ${ }^{238} \mathrm{U}$. A microscopic approach is used, since no adjustable parameters are involved. Cross section are expressed as a sum of DWBA transition amplitudes computed with the microscopic two-body interaction M3Y. Final target states are expressed as particle-hole excitations built from single particle states obtained within the HF+BCS theory applied with Skyrme force.
\end{abstract}

\section{Introduction}

It is well-known that the double differential neutron emission cross section coming from the scattering of a neutron on a target in its ground state can only be understood if one takes into account at least three different reaction processes, namely the direct process, the preequilibrium emission and the formation and evaporation of a compound nucleus. One has to also take into account the neutron emissions coming from the fission if the target is a fissionable nucleus, such as the actinides ${ }^{232} \mathrm{Th}$ and ${ }^{238} \mathrm{U}$. We focus here on the preequilibrium MSD process, which can be reduced to the first step of Feshbach, Kerman, Koonin theory (FKK) [1] since we are dealing with incident nucleon energy below $20 \mathrm{MeV}$. Such calculations have already been done within a semi-phenomenological approach [2] as the interaction used to generate the transitions is a simple central interaction with a Yukawa form factor and a strength adjusted to reproduce neutron emission data. Therefore, to avoid this phenomenological adjustment, a fully microscopic calculation is needed especially for nuclei where experimental data are scarce. In that case no reliable extrapolation could be made in applications where neutron cross section evaluations play a fundamental role, such as neutron moderation in the reactors. Though such a microscopic approach needs huge computing capacities, they are feasible since cluster computers have become very common.

\section{MSD cross section}

The MSD cross section can be evaluated within the distorted wave Born approximation (DWBA). For an inelastic scattering of an incident neutron of momentum $\mathbf{k}_{i}$ on a target nucleus in its ground state, the one-step MSD double-differential cross section is expressed as

$$
\begin{aligned}
\frac{d^{2} \sigma\left(\mathbf{k}_{i}, \mathbf{k}_{f}\right)}{d \Omega d E}= & \frac{1}{2 \Delta} \int_{E_{k_{f}}-\Delta}^{E_{k_{f}}+\Delta} d E_{k} \frac{\mu^{2}}{\left(2 \pi \hbar^{2}\right)^{2}} \frac{k_{f}}{k_{i}} \\
& \times \sum_{F} \frac{1}{2} \frac{\Gamma_{n}}{\left(E_{k_{i}}-E_{k}-E_{n}\right)^{2}+\frac{\Gamma_{n}^{2}}{4}}\left|T_{f \leftarrow i}^{(1)}\right|^{2},
\end{aligned}
$$

where the first order transition amplitude $T^{(1)}$ is

$$
T_{f \leftarrow i}^{(1)}=\left\langle\chi^{(-)}\left(\mathbf{k}_{f}\right), n J M \Pi\left|V_{e f f}\right| \chi^{(+)}\left(\mathbf{k}_{i}\right), 0\right\rangle .
$$

The functions $\chi^{(+)}\left(\mathbf{k}_{i}\right)$ and $\chi^{(-)}\left(\mathbf{k}_{f}\right)$ represent the distorted waves in the entrance and exit channels, respectively. The states $|0\rangle$ and $|n J M \Pi\rangle$ represent the target nucleus in its ground state and an excited state of angular momentum $J$, projection $M$, parity $\Pi$ and excitation energy $E_{n}$. As we deal with a noncorrelated ground state, the two-body residual interaction can only connect the $\mathrm{HF}+\mathrm{BCS}$ ground state to the excited states of one particle one hole structure.

As we use a crude description of the excited state of the nucleus, these states decay quickly towards more complicated states. This process is accounted for by the spreading width $\Gamma_{n}$ which is the origin of the Lorentzian spreading function $\frac{1}{2} \frac{\Gamma_{n}}{\left(E_{k_{i}}-E_{k}-E_{n}\right)^{2}+\frac{\Gamma_{n}^{2}}{4}}$ in (1). The widths $\Gamma_{n}$ can also contain the effect of the residual interaction between the projectile and the target which perturbs the nucleus level scheme before the transition. However as we do not have any microscopic calculation of these widths, we take the reasonable constant value of $2 \mathrm{MeV}$. Our results are not so sensitive to this value since an energy average is also necessary on both the neutron incoming and outgoing energies. This energy average is taken into account in (1) where we give a value of $1 \mathrm{MeV}$ to the width $\Delta$.

\section{Excited state of a deformed nucleus}

The final states of the target are particle-hole excitations constructed from single particle orbitals in the intrinsic frame. These orbitals have been calculated within the HF+BCS framework which uses the $\mathrm{SkM}^{*}$ Skyrme interaction. In the $\mathrm{HF}+\mathrm{BCS}$ theory, the ground state of an even-even nucleus is expressed in the intrinsic frame as

$$
\left|\varphi_{0}\right\rangle=\prod_{k>0}\left(u_{k}+v_{k} a_{k}^{+} a_{\bar{k}}^{+}\right)|-\rangle,
$$


where $|-\rangle$ is the particle vacuum and $a_{k}^{+}$is the single particle state creation operator. With the assumption of an axially symmetric nucleus in the intrinsic frame, the Skyrme Hartree-Fock Hamiltonian is solved in the cylindrical harmonic oscillator basis $\left\{n_{z} n_{\perp} \Lambda \Sigma\right\}$

$$
|k\rangle=\sum_{n_{z} \geq 0} \sum_{n_{\perp} \geq 0} \sum_{\Sigma= \pm \frac{1}{2}} c_{n_{z} n_{\perp} \Lambda_{k} \Sigma}^{(k)}\left|n_{z} n_{\perp} \Lambda_{k} \Sigma\right\rangle
$$

All details on this calculation can be found in $[3,4]$. The particle-hole excitations are expressed in the intrinsic frame as

$$
|p h\rangle=v_{p}^{2} v_{h}^{2} a_{p}^{+} a_{h}\left|\varphi_{0}\right\rangle
$$

The single particle state $a_{i}^{+}$are obtained from an axially symmetric calculation which preserves the symmetry with respect to the time reversal. Therefore, they are eigenfunctions of the projection $K$ of the angular momentum $J$ on the symmetry axis. Since we want to express the excited states in the laboratory frame with good total angular momentum, we use the projection of single-particle states on a spherical basis of harmonic oscillator wave functions. The cylindrical basis $\left\{n_{z} n_{\perp} \Lambda \Sigma\right\}$ is expanded in the spherical harmonic oscillator basis $|n l j K\rangle$. One can find all the details of this procedure in [5]. The result of this projection reads

$$
\left|k \Omega_{k}\right\rangle=\sum_{n \geq 0} \sum_{l \geq 0} \sum_{j=l-\frac{1}{2}}^{j=l+\frac{1}{2}} S_{n l j}^{(k)}\left|n l j \Omega_{k}\right\rangle=\sum_{l \geq 0} \sum_{j=l-\frac{1}{2}}^{j=l+\frac{1}{2}} C_{l j}^{(k)}\left|k^{\prime} l j \Omega_{k}\right\rangle
$$

where we have defined the single particle states

$$
\left|k^{\prime} l j \Omega_{k}\right\rangle=\sum_{n} S_{n l j}^{(k)}\left|n l j \Omega_{k}\right\rangle=b_{k l j \Omega_{k}}^{+}|-\rangle
$$

The particle-hole states in the laboratory frame are then expressed as:

$$
|n J M \Pi\rangle=C_{l_{p} j_{p} \Omega_{p}}^{(p)} C_{l_{h} j_{h} \Omega_{h}}^{(h)}\left[b_{p l_{p} j_{p}}^{+} \otimes b_{h l_{h} j_{h}}^{+}\right]_{M}^{J \Pi}\left|\phi_{0,0}^{(0)}\right\rangle .
$$

The state $\left|\phi_{0,0}^{(0)}\right\rangle$ represents the ground state projected from $\left|\varphi^{(0)}\right\rangle$, namely:

$$
\left|\phi_{0,0}^{(0)}\right\rangle=\frac{1}{8 \pi^{2}} \int d \Theta D_{0,0}^{0}{ }^{*}(\Theta) R(\Theta)\left|\varphi^{(0)}\right\rangle .
$$

The contraction of the two-body interaction can be expressed in terms of the one-body transition matrix element between the ground and excited states. Thus, if one writes the two-body interaction in the second quantification

$$
\hat{V}_{e f f}=\frac{1}{4} \sum_{\alpha \beta \gamma \delta}\left\langle\alpha \beta\left|\hat{V}_{e} f f\right| \widetilde{\gamma \delta}\right\rangle a_{\alpha}^{+} a_{\beta}^{+} a_{\delta} a_{\gamma}
$$

two single particle operators are contracted between the projectile incoming and outgoing wave functions, and the two others are contracted between the target ground- and excited states

$$
\left\langle n J M \Pi\left|b_{\alpha}^{+} b_{\gamma}\right| \phi_{0,0}^{(0)}\right\rangle \simeq C_{l_{p} j_{p} \Omega_{p}}^{(p)} C_{l_{h} j_{h} \Omega_{h}}^{(h)} v_{p \Omega_{p}} v_{h \Omega_{h}}
$$

where the single-particle occupancies are calculated in the BCS approximation.

\subsection{Results for inelastic neutron scattering on ${ }^{208} \mathrm{~Pb}$}

We have first tested our approach for neutron inelastic scattering on ${ }^{208} \mathrm{~Pb}$. In particular, it is a good test of the M3Y interaction [6] which is used to generate the transitions between the target ground and excited states. We compute the MSD cross section using RPA excited states coming from a HF+RPA calculation with the D1S Gogny force [7-9]. The good results previously obtained for proton inelastic scattering between 40 and $200 \mathrm{MeV}$ let us assume that the RPA wave functions are accurate and can be used with confidence in scattering calculation [10]. All the DWBA cross sections are computed with the DWBA98 code [11]. The distorted waves in the entrance and exit channels are obtained with the KoningDelaroche optical potential [12].

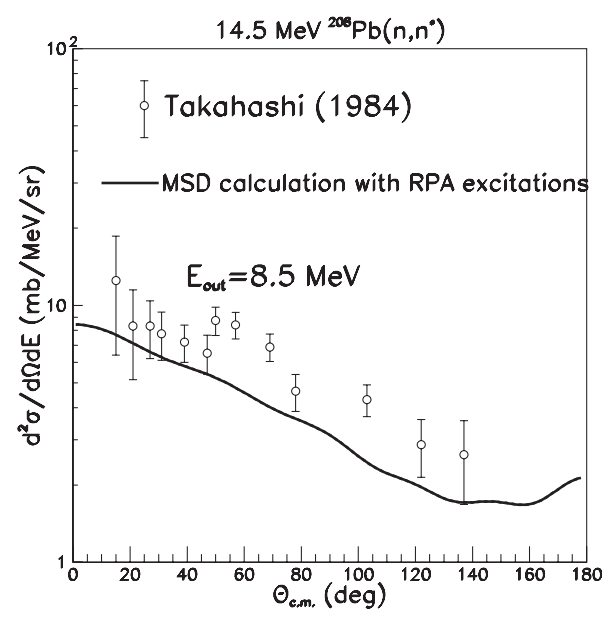

Fig. 1. Comparison between neutron emission experimental data and the calculated MSD double-differential cross sections for $14.5 \mathrm{MeV}$ neutron scattering on ${ }^{208} \mathrm{~Pb}$. The solid line is the one-step MSD cross section obtained with the M3Y two-body interaction and the RPA excited states obtained form the HF+RPA/D1S calculation.

We show in figure 1 the double differential cross section corresponding to inelastic scattering of $14.5 \mathrm{MeV}$ on ${ }^{208} \mathrm{~Pb}$ and an outgoing neutron energy of $8.5 \mathrm{MeV}$. Our result is in fair agreement with the expereimental data [13], even if there seems to be a discrepancy at intermediate angles. This feature may come from the contribution of some collective states whose contributions are averaged in our cross section calculation. However, this result makes us believe that the M3Y interaction is accurate enough for this kind of MSD calculation.

\subsection{Results for inelastic neutron scattering on ${ }^{238} \mathrm{U}$}

In this section we display the results obtained for neutron inelastic scattering on ${ }^{238} \mathrm{U}$. The MSD cross sections (1) are calculated with the microscopic M3Y interaction and the particle-hole excited states wave functions and energies have been obtained within the $\mathrm{HF}+\mathrm{BCS}$ framework with the $\mathrm{SkM}^{*}$ force in a basis whose size is up to $20 \hbar \omega$ to ensure convergence. Even in the case of such a strongly deformed 

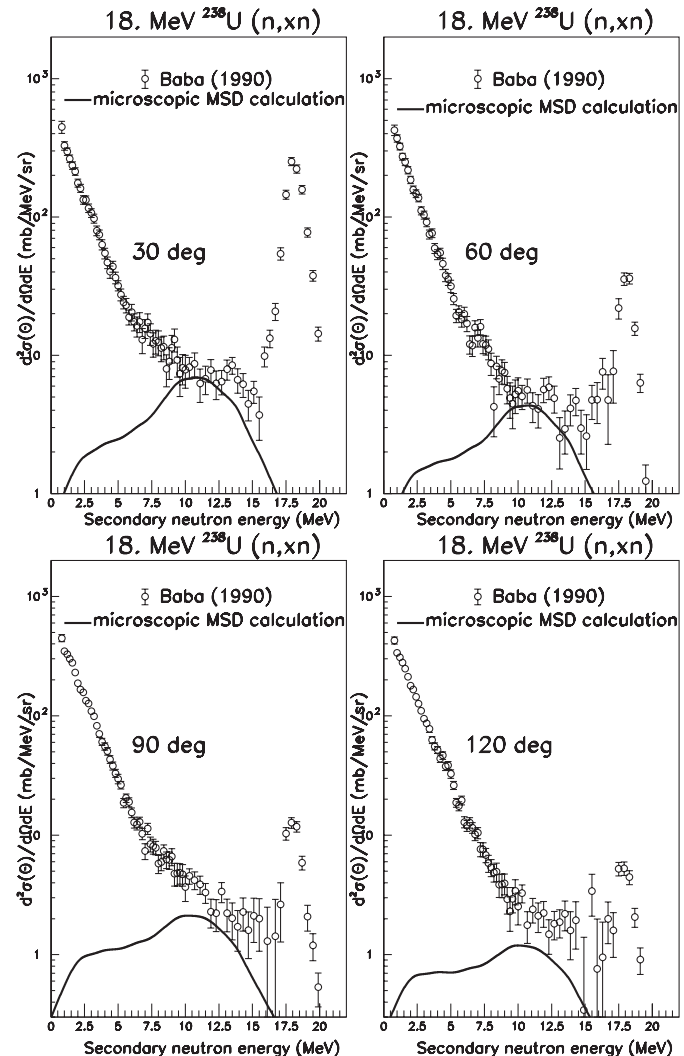

Fig. 2. Comparison of the calculated MSD double-differential cross sections for ${ }^{238} \mathrm{U}$ at $18 \mathrm{MeV}$ with the experimental data at four different angles $\left(30^{\circ}, 60^{\circ}, 90^{\circ}\right.$ and $\left.120^{\circ}\right)$. The solid line is the one-step MSD cross section obtained with the M3Y two-body interaction and the particle-hole states obtained form the $\mathrm{HF}+\mathrm{BCS} \mathrm{SkM}{ }^{*}$ calculation.

Table 1. Parameters of the optical potential used to compute the distorted waves in both entrance and exit channels.

\begin{tabular}{lll}
\hline Strength MeV & Radius fm & Diffuseness fm \\
\hline$V(E)=44.47-0.2998 E$ & $r_{V}=1.249$ & $\alpha_{V}=0.660$ \\
$W_{S}(E)=5.506+0.33 E$ & $r_{W S}=1.208$ & $\alpha_{W S}=0.614$ \\
$W_{V}(E)=0.06613+0.05 E$ & $r_{W V}=1.248$ & $\alpha_{W V}=0.594$ \\
$V_{S O}=5.702$ & $r_{V S O}=1.121$ & $\alpha_{V S O}=0.590$ \\
\hline
\end{tabular}

target, the excited states of the target in the energy region of interest present a quasi-continuous structure and one can expect the effect of the coupled channels to be averaged and therefore to only make a slight difference on cross sections evaluation. However, such a statement has to be checked but this is beyond the scope of the present work.

The distorted waves in both entrance and exit channels are obtained from a spherical calculation using a local energy dependent optical potential $U(r, E)$ adjusted to reproduce the diagonal S-matrix elements corresponding to the coupled channels calculations. The parameters of this optical potential are given in table 1 where the symbols are given in a conventional manner.

Again, the DWBA98 code is used to compute all the DWBA cross sections, but it has been parallelized since our
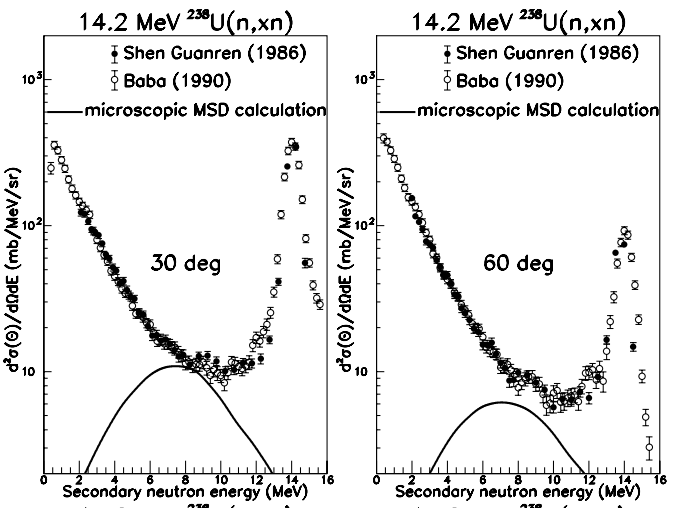
$14.2 \mathrm{MeV}^{238} \mathrm{U}(\mathrm{n}, \mathrm{xn})$
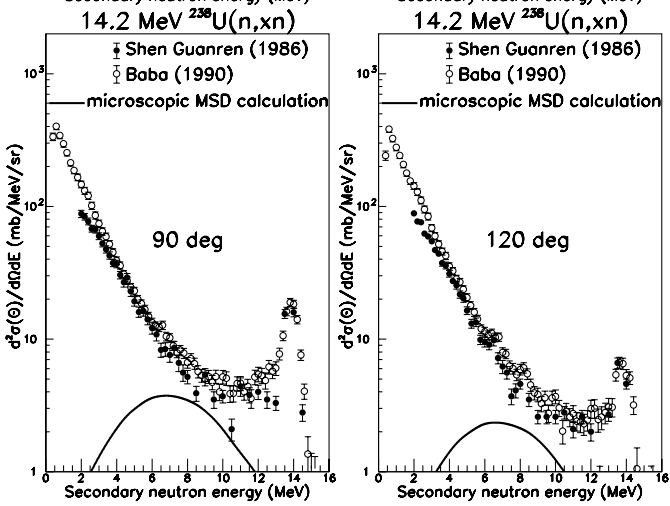

Fig. 3. Same comparisons for $14.2 \mathrm{MeV}$ incident neutron. See figure 2 caption.

Table 2. Number of particle-hole configurations in ${ }^{238} \mathrm{U}$ involved for our MSD cross section calculation for different neutron incident energies and associate computing time for calculations on 42 processors (type AMD $2 \mathrm{GHz}$ Opteron).

\begin{tabular}{ccc}
\hline $\begin{array}{c}\text { Incident } \\
\text { energy }(\mathrm{MeV})\end{array}$ & $\begin{array}{c}\text { Number of ph } \\
\text { configurations }\end{array}$ & $\begin{array}{c}\text { Computation } \\
\text { time }\end{array}$ \\
\hline 11.8 & $1,873,916$ & $\simeq 1 \mathrm{~h} 42 \mathrm{~min}$ \\
14.1 & $2,756,855$ & $\simeq 2 \mathrm{~h} 22 \mathrm{~min}$ \\
18. & $5,217,220$ & $\simeq 4 \mathrm{~h} 29 \mathrm{~min}$ \\
\hline
\end{tabular}

calculations involves the knowledge of several millions of transition matrix elements. As an illustration, we depict in table 2 the number of particle-hole configurations we have to take into account and the DWBA calculation times required.

We display on figures 2, 3 and 4 comparisons between our MSD calculations and experimental data for $18 . \mathrm{MeV}[14,15]$, 14.1 MeV [14-16] and $11.8 \mathrm{MeV}$ [17] neutron scattering on ${ }^{238} \mathrm{U}$ at four different angles of emission.

The distributions show a good behaviour both in magnitude and angular shape and are consistent with previous MSD cross sections obtained within more phenomenological approach [2]. We have to emphasize that these results have been calculated without any adjustable parameters. The MSD cross sections are underestimated at high emission energy. This discrepancy has already been noticed by Miura et al. [17] who make an assumption of possible existence of collective discrete levels at excitation energies between 1.5 and $4 \mathrm{MeV}$. Our microscopic calculation still has the same problem, 

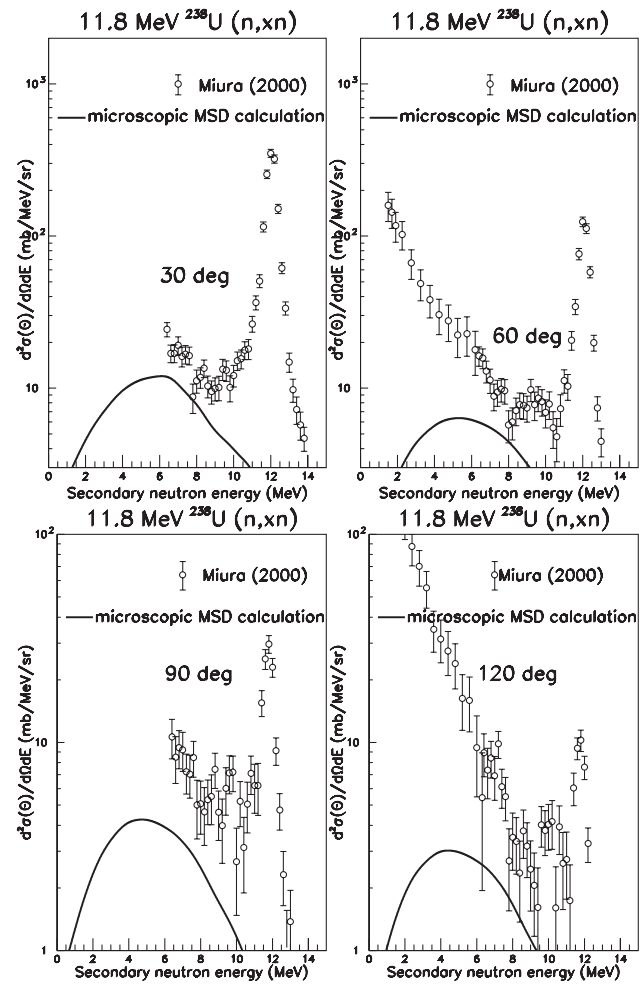

Fig. 4. Same comparisons for $11.8 \mathrm{MeV}$ incident neutron. See figure 2 caption.

however this problem might be solved by taking into account the shift in single particle energy which may appear if one applies properly the projection method to express particle-hole excitation energies in the laboratory frame while our calculations use the energies obtained in the intrinsic frame [18]. This calculation could show a compression of the single particle energies around the Fermi surface and, as a consequence, should increase the MSD cross section contributions at high emission energy.

\section{Conclusion}

Microscopic calculation of MSD component without any parameter adjustment has been done for medium neutron scattering on ${ }^{208} \mathrm{~Pb}$ and ${ }^{238} \mathrm{U}$. The results are in good agreement with the experimental data. The calculation time induced is quite affordable and proves that microscopic approaches are realizable for this type of calculation and could be easily extended to assess the validity of our results. These calculations have to be extended to other targets, such as ${ }^{232} \mathrm{Th}$ and other deformed nuclei for which data are available. However, several verifications have to be performed such as the studies of the MSD cross section sensibility to the two-body interaction. One possibility is to use the Gogny's finite range interaction D1S [19] as a residual interaction. Even if this interaction has been fitted for nuclear structure and not for scattering processes, it gives very good estimation of giant resonances in closed-shell nuclei. As these resonances lay above $10 \mathrm{MeV}$, the use of the Gogny interaction seems to be reasonable for incident nucleon energy below $20 \mathrm{MeV}$. These calculations are in progress. Another improvement would be to use a more accurate descriptions of the target excited states, such as HF-Bogoliubov wave functions obtained with a finite range two-body interaction.

We thank Prof. A. Kerman for valuable discussions. This work was carried out under the auspices of the National Nuclear Security Administration of the US Department of Energy at Los Alamos National Laboratory under Contract No. DE-AC52-06NA25396.

\section{References}

1. H. Feshbach, A. Kerman, A.K. Koonin, Ann. Phys. (NY) 125, 429 (1980).

2. T. Kawano, T. Ohsawa, M. Baba, T. Nakagawa, Phys. Rev. C 63, 034601 (2001).

3. D. Vautherin, Phys. Rev. C 7, 296 (1973).

4. H. Flocard, P. Quentin, A. Kerman, D. Vautherin, Nucl. Phys. A 203, 443 (1973).

5. L. Bonneau, T. Kawano, T. Watanabe, S. Chiba, Phys. Rev. C (to be published).

6. N. Anantaraman, H. Toki, G.F. Bertsch, Nucl. Phys. A 398, 269 (1983).

7. J.P. Blaizot, D. Gogny, Nucl. Phys. A 284, 429 (1977).

8. J. Dechargé, L. Šips, Nucl. Phys. A 407, 1 (1983).

9. D. Gogny, J. Dechargé, Journal de Physique C 4, 221 (1984).

10. M. Dupuis, S. Karataglidis, E. Bauge, J.P. Delaroche, D. Gogny, in Workshop proceedings, Perspectives on Nuclear Data for the Next Decade, Bruyères-le-Châtel, France, 26-28 Sept. 2005, edited by E. Bauge (OECD Publishing, 2006), p. 67.

11. J. Raynal, Computer code DWBA98, NEA 1209/05 (1998).

12. A.J. Koning, J.P. Delaroche, Nucl. Phys. A 713, 231 (2003).

13. A. Takahashi, M. Fukasawa, Y. Yanagi, J. Yamamoto, J. Nucl. Sci. Technol. 21, 577 (1984)

14. M. Baba, H. Wakabayashi, N. Ito, K. Maeda, N. Irakawa, J. Nucl. Sci. Technol. 27, 601 (1990).

15. M. Baba, S. Matsuyama, N. Ito, K. Maeda, N. Irakawa, in Proceedings of International Conference on Nuclear Data for Science and Technology, Jülich, Germany, 1991, edited by S.M. Qaim (Springer-Verlag, Berlin/Heidelberg, 1992), p. 349.

16. Shen Guanren et al., Chinese Physics 8, 1000 (1988).

17. T. Miura, M. Baba, M. Ibaraki et al., Ann. Nucl. Energy 28, 937 (2000).

18. A. Kerman (private communication).

19. J.F. Berger, M. Girod, D. Gogny, Comput. Phys. Commun. 159, 365 (1991). 